\title{
Neutrino Oscillation Parameters: Future
}

\section{J. Lopez-Pavon}

Instituto de Física Corpuscular, Universidad de Valencia and CSIC, Edificio Institutos

Investigación, Catedrático José Beltrán 2, 46980 Spain

E-mail: jacobo.lopez@uv.es

\section{E. Worcester}

Brookhaven National Laboratory

Upton, NY, USA

E-mail: etw@bnl.gov

The Neutrino Oscillation Workshop (NOW2018) included a session addressing neutrino oscillation parameters in the future. This session included discussion of planned and proposed experiments, detector technologies, and analysis techniques to better measure neutrino oscillation parameters. Constraint of systematic uncertainty in future precision measurements was a topic of particular interest. This proceedings is a brief summary of the presentations in the Oscillation Parameters: Future session; readers are directed to individual contributions to the proceedings for more detailed information.

Neutrino Oscillation Workshop (NOW2018)

9 - 16 September, 2018

Rosa Marina (Ostuni, Brindisi, Italy) 


\section{Introduction}

Measurements of the parameters governing neutrino oscillation are quite mature, with most of the neutrino mass splittings $\left(\left|\Delta m_{32}^{2}\right|, \Delta m_{21}^{2}\right)$ and mixing angles $\left(\theta_{13}, \theta_{23}\right.$, and $\left.\theta_{12}\right)$ known to within $3 \%[1,2,3]$. The $\mathrm{CP}$ violating parameter, $\delta_{C P}$, is not yet well measured, though global fits to neutrino oscillation measurements are beginning to provide some information about its value, based in particular on results from $\mathrm{T} 2 \mathrm{~K}$ and NOvA. The main goals of future oscillation parameter measurements are to resolve the neutrino mass ordering ambiguity, determine whether $\mathrm{CP}$ symmetry is violated in neutrino mixing (i.e., whether $\delta_{C P}$ is not equal to zero or pi), determine the octant of $\theta_{23}$, and make precision measurements of oscillation parameters in order to test the unitarity of the PMNS matrix, to faciliate comparison to theoretical models, and to provide sensitivity to physics beyond the Standard Model.

The session on neutrino oscillation parameters in the future consisted of contributions describing planned or proposed experiments, detector technologies, and theoretical studies of neutrino mixing. Contributions included discussion of oscillation parameter measurements themselves, but also discussion of calculations, measurements, and analysis techniques designed to constrain systematic uncertainties in future oscillation parameter measurements; this topic was a significant focus of the session as it is clear that the ability to understand and constrain systematic uncertainty is a critical aspect of any precision oscillation measurement program. Several contributions also discussed physics beyond the Standard Model and how the presence of such new physics could impact future oscillation parameter measurements. The following sections briefly describe the theoretical and experimental contributions to the session. While the topics are separated in this way for convenience, there is significant overlap in content and close collaboration between theory and experiment is required to make the most of future oscillation measurements. We refer the reader to individual proceedings for all details; in this document we attempt only a brief summary of each presentation in the session.

\section{Theory}

Global fits to neutrino oscillation data [1,2,3] show a consistent picture of the three neutrino framework which will be completed in the future with the measurement of the Dirac CP phase $\delta$, the octant of $\theta_{23}$ and the determination of the neutrino ordering. Experiments as KATRIN or cosmological probes will also be sensitive to the absolute neutrino mass scale which is not accessible through neutrino oscillation experiments. However, the origin and nature (Majorana or Dirac) of neutrino masses remains an open question in the Standard Model (SM). The minimal models, typeI, type-II and type-III seesaws, do not involve the extension of the SM symmetries and generate the masses at tree level. Other attractive options imply the addition of extra gauge symmetries, as in the Left-Right symmetric models, or induce neutrino masses at radiative level. The canonical realizations of models originally motivated by Grand Unification Theories (GUT) imply very heavy new physics scales making extremely difficult to test them. Nevertheless, symmetry protected scenarios as the inverse or direct seesaws are technically natural realizations with neutrino mass mediators at the electroweak scale. The heavy states can thus be produced and searched for in beam dump experiments and colliders. Furthermore, neutrinoless double beta decay experiments will allow us 
to test if neutrinos are Dirac or Majorana particles. This is a very relevant issue: on one hand, because the observation of this process would imply that lepton number is violated and, on the other hand, because it will allow to discriminate among neutrino mass models.

Notice that the simplest model, a Type-I seesaw where the mass mediators are right-handed neutrinos (sterile neutrinos), can accommodate the observed neutrino masses and mixing with a new physics scale $M$, determined by the mass of the new states, from the $\mathrm{eV}$ to the GUT scale. Two different phenomenological regimes can be distinguished depending on the mass of the righthanded neutrinos $M$ : i) $M$ heavier than the electroweak scale (right-handed neutrinos integrated out from low energy spectrum); ii) $M \lesssim 1 \mathrm{keV}$ for which the new states can be kinematically produced in the neutrino oscillation source via mixing. Both cases induce deviations from the unitarity of the $3 \times 3$ PMNS matrix, but the bounds applying to these deviations are qualitatively different. The global bounds in both regimes and the future sensitivity of DUNE and IceCube to these new physics effects for masses $M \gtrsim 10 \mathrm{eV}$ was presented in [4].

The neutrino sector is also deeply linked to the mysterious flavour puzzle, maybe holding the key to its solution. The goal in this context is to explain the lepton and quark mixing patterns with the addition of extra flavor symmetries. These underlying discrete symmetries induce correlations among the neutrino mixing parameters that can be tested in neutrino oscillation experiments opening a window to falsify the different models. In [5] a phenomenological analysis of the potential of DUNE and T2HK to falsify these models and a detailed discussion regarding the experimental precision required was presented.

Neutrino masses suggest the existence of fundamental new physics which may also shed light on other SM open questions as the Dark Matter (DM) problem. Several interesting possibilities for Dark Matter candidates connected to the neutrino mass problem were presented in [6].

Indeed, all these aspects of neutrino theory briefly described above were nicely reviewed in the first plenary talk of the session by Jose W F Valle [6].

Regarding neutrino oscillation experiments, one of the most relevant issues in future precision facilities as DUNE or T2HK, given the excellent statistics available in these experiments, is the role of systematic uncertainties. A careful control of systematics is a fundamental requirement in order to achieve the high precision goal for the determination of neutrino mixing parameters, in particular through appearance channels. The near detectors will allow to mitigate this problem but not completely eliminate the dependence on the nuclear interaction models. This was one of the main topics of the session. Nuclear interaction models will play an extremely relevant role in this context. As presented in [7,8], a theoretical effort from the nuclear theory side is required in order to improve the nuclear model predictions which still present important inconsistencies with existing data. An experimental answer to this problem with a precise measurement of neutrino cross sections would likely require dedicated facilities as the proposed ENUBET [20] or NuSTORM [10], in particular regarding electron neutrino cross sections. To illustrate the impact of systematics on the mixing parameters determination by future long baseline neutrino oscillation facilities, a phenomenological study was presented by Osamu Yasuda [11].

Future Near detector facilities also provide a laboratory for the search of beyond the SM particles. This type of searches include $0.1-1 \mathrm{GeV}$ scale right-handed neutrinos, new neutrino interactions, dark matter or millicharged particles. A particularly interesting process that could potentially be searched for in these detectors is the neutrino trident. Notice that models involving 
$Z^{\prime}$ gauge bosons as the proposed to explain the flavor anomalies can induce this process. In [12] the sensitivity of near detectors to the neutrino trident production was nicely discussed.

\section{Experiment}

The experimental presentations described plans for future high-precision measurements of oscillation parameters in the three-neutrino paradigm, sensitivity of future experiments to additional neutrinos and other beyond-the-Standard-Model effects, experiments designed to constrain systematic uncertainties, in particular from uncertainties in neutrino flux and interaction modeling, and new accelerator and detector technologies applicable to neutrino physics.

Plans for precision oscillation parameter measurements in the three-flavor paradigm center around three next-generation experiments; JUNO, DUNE, and Hyper-K all anticipate a start of data collection in the coming decade.

The Jiangmen Underground Neutrino Observatory (JUNO) is a 20-kt liquid scintillator detector located in Guangdong, China, 53-km from a set of high power nuclear reactors. This "medium baseline" location facilitates precise measurements of $\theta_{12}, \Delta m_{21}^{2}$, and $\Delta m_{e e}^{2}$, with an anticipated precision of better than $1 \%$ for all three measurements. JUNO is also expected to be sensitive to the neutrino mass ordering via differences in the positron spectrum between normal and inverted ordering. JUNO is currently under construction and expects to begin collecting data in 2021. In addition to the physics sensitivity, the JUNO presentation [13] provided details on the civil construction and R\&D progress and design plans for the acrylic vessel, PMTs, scintillator production, readout electronics, and calibration schemes.

The Deep Underground Neutrino Experiment (DUNE) is composed of a 40-kt (fiducial) liquid argon TPC far detector at Sanford Underground Research Facility (SURF) in South Dakota, US, located at a baseline of $1300 \mathrm{~km}$ from a neutrino beam and near detector complex at Fermilab. DUNE scientific goals include precise measurements of $v_{\mu} \rightarrow v_{e}$ and $v_{\mu} \rightarrow v_{\mu}$ oscillation, providing sensitivity to $\mathrm{CP}$ violation, determination of the neutrino mass ordering, and precise measurements of neutrino mixing parameters $\Delta m_{32}^{2}, \theta_{13}, \theta_{23}$, and $\delta_{C P}$. Other scientific goals include sensitivity to baryon number violating processes, detection of neutrinos from core-collapse supernovae, and searches for physics beyond the standard model. In addition to the physics sensitivity of DUNE, the presentation [14] provided details of the design for single- and dual-phase LArTPC far detector modules and development of large-scale prototypes for both detector technologies hosted at CERN. A parallel presentation [15] described more details on the prototypes, called protoDUNEs, with a particular focus on the dual phase design and status.

Hyper-Kamiokande is a 190-kt (fiducial) water Cerenkov detector located in Kamioka, Japan, at a baseline of $250 \mathrm{~km}$ from the J-PARC neutrino beam and near detector complex. Hyper-K has a science program to search for $\mathrm{CP}$ violation and make precision oscillation parameter measurements using the neutrino beam, to determine the neutrino mass ordering and $\theta_{23}$ octant using atmospheric neutrinos, to search for nucleon decay, and to study solar neutrinos, neutrino astronomy and astrophysics. The presentation [16] discussed an option to add a second Hyper-K far detector in Korea, providing increased resolution on the measurement of $\delta_{C P}$. In addition to the physics sensitivity, PMT tests demonstrating improved single photon detection efficiency relative to Super-K and planned upgrades to the beam and near detector at J-PARC were shown. 
A presentation [17] describing neutrino oscillation measurements with atmospheric neutrinos focused on the KM3Net/ORCA and PINGU experiments. Km3Net/ORCA modules are deployed in the ocean; module deployment is ongoing and initial data from the first ORCA modules was shown. PINGU is a high density upgrade to IceCube in which 26 additional strings are added between the DeepCore strings. Sensitivity of both experiments to the neutrino mass ordering, atmospheric oscillation parameters, and $v_{\tau}$ appearance were shown.

The presentation [18] on the European Spallation Source neutrino Super Beam (ESSnuSB) described possible modifications to the ESS beam that would facilitate sensitivity to CP violation at the second oscillation maximum by producing a $5 \mathrm{MW}$ proton beam. The proposed far detector would be a 500-kt fiducial volume water Cherenkov detector located $540 \mathrm{~km}$ from the ESS at a depth of 1232 meters. The SNO+ presentation [19] described the status and physics program of SNO+, which includes nucleon decay and solar neutrino measurements in the water phase as well as measurements of $\Delta m_{21}^{2}$ using reactor antineutrinos and sensitivity to neutrinoless double beta decay in the scintillator phase.

The presentation from [8] described the importance of neutrino interaction modeling and measurements for determination of oscillation parameters and the current state of interaction measurements. Instances in which existing models of neutrino interactions are insufficient to describe experimental data were emphasized. Additionally, analysis techniques developed to better probe new models and break degeneracy among models were described.

Several experimental presentations addressed constraint of systematic uncertainty in future oscillation parameter measurements. ENUBET [20] is a proposed narrow band beam at the $\mathrm{GeV}$ scale designed to reduce flux uncertainties to the percent level, allowing precise measurements of neutrino cross sections. The short baseline and well-known flavor composition of the beam would also facilitate searches for non-standard interactions and sterile neutrinos. Details of beamline R\&D were shown. The MICE/nuStorm presentation [21] discussed the use of muon cooling to design a neutrino factory in which the flux uncertainty would be at the percent level. Results from the MICE experiment, designed to study factors that affect muon cooling, were shown. A description was provided of nuStorm, a proposed neutrino factory based on existing technology that could make precision measurement of neutrino interactions as well as search for sterile neutrino oscillations.

An idea for a new detector technology dubbed "Liquid O" was presented [22]. In this detector concept, liquid scintillator is produced in an opaque state, such that scintillation light may be observed as a function of time to produce TPC-like event images. This would significantly improve the particle identification capabilities of liquid scintillator detectors. The physics potential of such a detector for neutrinoless double beta decay searches was explored and first results from a prototype detector were shown.

While not officially in the future oscillation parameter measurements session, the workshop concluded with a compelling argument for the need for next-generation measurements of the solar mixing parameters [23], in which it was argued that improving these measurements is a critical aspect of a combined program of laboratory, cosmology, and astronomy neutrino science.

\section{Acknowledgements}

JLP acknowledges support by the "Generalitat Valenciana" (Spain) through the "plan GenT" 
program (CIDEGENT/2018/019).

\section{References}

[1] I. Esteban, M. C. Gonzalez-Garcia, A. Hernandez-Cabezudo, M. Maltoni and T. Schwetz, arXiv:1811.05487 [hep-ph].

[2] P. F. de Salas, D. V. Forero, C. A. Ternes, M. Tortola and J. W. F. Valle, Phys. Lett. B 782, 633 (2018) doi:10.1016/j.physletb.2018.06.019 [arXiv:1708.01186 [hep-ph]].

[3] F. Capozzi, E. Di Valentino, E. Lisi, A. Marrone, A. Melchiorri and A. Palazzo, Phys. Rev. D 95 , no. 9, 096014 (2017) doi:10.1103/PhysRevD.95.096014 [arXiv:1703.04471 [hep-ph]].

[4] Josu Hernandez-Garcia, these proceedings.

[5] Sanjib Kumar Agarwalla, these proceedings.

[6] J.W.F. Valle, these proceedings.

[7] Monireh Kabirnezhad, these proceedings.

[8] Callum Wilkinson, these proceedings.

[9] Fabio Pupilli, these proceedings.

[10] Maurizio Bonesini, these proceedings.

[11] Osamu Yasuda, these proceedings.

[12] Matheus Hostert, these proceedings.

[13] Gioacchino Ranucci, these proceedings.

[14] Nuno Barros, these proceedings.

[15] Andrea Scarpelli, these proceedings.

[16] Yury Kudenko, these proceedings.

[17] Dorothea Samtleben, these proceedings.

[18] Tord Ekelof, these proceedings.

[19] Edward Leming, these proceedings.

[20] Fabio Pupilli, these proceedings.

[21] Maurizio Bonesini, these proceedings.

[22] Anatael Cabrera, these proceedings.

[23] John Beacom, these proceedings. 